\title{
A physical explanation for the magnetic decrease ahead of dipolarization fronts
}

\author{
Z. H. Yao ${ }^{1}$, J. Liu ${ }^{2}$, C. J. Owen ${ }^{1}$, C. Forsyth ${ }^{1}$, I. J. Rae ${ }^{1}$, Z. Y. Pu ${ }^{3}$, H. S. Fu ${ }^{4}$, X.-Z. Zhou ${ }^{3}$, Q. Q. Shi ${ }^{5}$, A. M. Du ${ }^{6}$, \\ R. L. Guo ${ }^{3}$, and X. N. Chu ${ }^{2}$ \\ ${ }^{1}$ UCL Mullard Space Science Laboratory, Dorking, RH5 6NT, UK \\ ${ }^{2}$ Department of Earth, Planetary, and Space Sciences, University of California, \\ Los Angeles, CA 90095-1567, USA \\ ${ }^{3}$ School of Earth and Space Sciences, Peking University, Beijing 100871, China \\ ${ }^{4}$ Space Science Institute, School of Astronautics, Beihang University, Beijing 100191, China \\ ${ }^{5}$ Shandong Provincial Key Laboratory of Optical Astronomy and Solar-Terrestrial Environment, School of \\ Space Science and Physics, Shandong University, Weihai 264209, China \\ ${ }^{6}$ Institute of Geology and Geophysics, Chinese Academy of Sciences, Beijing 100029, China
}

Correspondence to: Z. H. Yao (z.yao@ucl.ac.uk)

Received: 26 June 2015 - Revised: 13 September 2015 - Accepted: 8 October 2015 - Published: 26 October 2015

\begin{abstract}
Recent studies have shown that the ambient plasma in the near-Earth magnetotail can be compressed by the arrival of a dipolarization front (DF). In this paper we study the variations in the characteristics of currents flowing in this compressed region ahead of the DF, particularly the changes in the cross-tail current, using observations from the THEMIS satellites. Since we do not know whether the changes in the cross-tail current lead to a field-aligned current formation or just form a current loop in the magnetosphere, we thus use redistribution to represent these changes of local current density. We found that (1) the redistribution of the cross-tail current is a common feature preceding DFs; (2) the redistribution of cross-tail current is caused by plasma pressure gradient ahead of the DF and (3) the resultant net current redistributed by a DF is an order of magnitude smaller than the typical total current associated with a moderate substorm current wedge (SCW). Moreover, our results also suggest that the redistributed current ahead of the DF is closed by currents on the DF itself, forming a closed current loop around peaks in plasma pressure, what is traditionally referred to as a banana current.
\end{abstract}

Keywords. Magnetospheric physics (current systems; magnetotail; plasma sheet)

\section{Introduction}

Bursty bulk flows (BBFs) are intervals of fast Earthward plasma and magnetic flux transport in the plasma sheet, and are usually considered the most important carriers of mass and energy towards the near-Earth region (Angelopoulos et al., 1992, 1994). A BBF consists of one or more individual flow bursts (FBs) (Angelopoulos et al., 1992), also referred to as dipolarizing flux bundles (DFBs) (Liu et al., 2013a, b). DFB emphasizes magnetic perturbation signatures, whereas FB describe mass and magnetic flux transport. Both the plasma velocity and the north-south component of the magnetotail's magnetic field inside the BBF are significantly larger than in the surrounding region. They carry a stronger magnetic field and current density on their leading edge than in the surrounding magnetotail (Liu et al., 2013a, 2014). The front of the DFB is often associated with a sharp increase in the northward magnetic field component $B_{z}$ and is thus known as the dipolarization front (DF) (Nakamura et al., 2002; Sergeev et al., 2009). This is usually a kineticscale structure of width of the order of an ion gyro-radius, i.e. $\sim 1000 \mathrm{~km}$, and is often associated with particle energization and wave activity (Fu et al., 2011; Tang et al., 2013; Zhang and Angelopoulos, 2014; Zhou et al., 2009). The formation of the DF structure may be associated with a magnetic reconnection process occurring in the deeper tail (Angelopoulos et 
al., 2013; Forsyth et al., 2008; Fu et al., 2013; Sitnov et al., 2013; Vogiatzis et al., 2015). Analogous DF-like structures have also been observed at Mercury and Jupiter (Kronberg et al., 2005; Sundberg et al., 2012; Sun et al., 2015), suggesting that this structure may be common in all planetary magnetotails.

Previous studies have suggested an SCW comprises a number of "wedgelet" structures (Rostoker, 1991), which have recently been suggested to be associated with the DFs in the magnetotail (Liu et al., 2013a). Individual FBs within the $\mathrm{BBF}$ are associated with field-aligned currents (FACs) that flow into the ionosphere on their dawnward edge and out of the ionosphere on their duskward edge, which forms a wedgelet (Birn and Hesse, 1996; Forsyth et al., 2008; Sergeev et al., 1996). Both simulations (Birn and Hesse, 2013; Birn et al., 1999) and observations (Yao et al., 2012, 2014a) have shown that the braking of BBFs in the nearEarth magnetotail may lead to current disruption and the formation of the FACs necessary to support an SCW. However, Forsyth et al. (2014) showed that the azimuthal structure of the SCW observed at low altitudes was not consistent with previous observations of BBF's FACs. Very recent results suggest that the wedgelet has dawn-dusk asymmetry (Liu et al., 2015), which might be a solution for this inconsistency. More studies are needed to reveal the relation between wedgelets and substorm current wedge.

Magnetic perturbations ahead of BBFs were studied by Ohtani et al. (2004). Their study shows that the northsouth component magnetic field decreases ahead of the front boundary of BBF. They suggested that this magnetic decrease might be a consequence of a remnant feature of a flux rope (Slavin et al., 2003). Slavin et al. (2003) also noted that the ambient plasma was compressed ahead of BBFs. Yao et al. (2014b) suggested this compression may trigger a pseudobreakup, rather than a fully developed substorm. Simulations have suggested that the magnetic perturbation ahead of the BBF is caused by the reflected ions from the DF (Zhou et al., 2014). However, the secondary dawnward current carried by the DF-reflected ions in the simulation is not a self-consistent system, especially since the secondary currents reduce the background magnetic field $B_{z}$ to very small or negative values. We thus need more in situ observations to build a selfconsistent physical connection between the current systems associated with the DF and the magnetic decrease ahead of the DF (Pan et al., 2015). In this paper we statistically examine the magnetic field and pressure signatures preceding DFs in order to evaluate the importance of a DF in modifying the near-Earth current system. Our analysis is based on a large database of THEMIS observations from the 2007 to 2011 tail seasons. Based on the magnetic field and pressure features in the compression region ahead of the DF, we then deduce the local redistribution of the cross-tail current, in order to determine the importance of this compression effect in modifying the more global magnetotail current system. Our study presents the compression effect ahead of DF, the consequent current system and the magnetic perturbations.

\section{Observations}

\subsection{Data set}

The THEMIS mission consists of five identical satellites, which provide extended periods of observations of the magnetotail (Angelopoulos, 2008). In this study, we use the magnetic field data from the fluxgate magnetometers (FGM) (Auster et al., 2008), particle data from the electrostatic analysers (ESA) (McFadden et al., 2008) and solid-state telescopes (SST) (Angelopoulos, 2008) on all five spacecraft. We use the combined $3 \mathrm{~s}$ resolution fast-survey ESA and SST flux measurements to calculate the plasma pressure and flow velocity. As the basis of our statistical study, we use the independently defined list of Earthward-travelling DFs from Liu et al. (2013a). This includes events identified from the measurements of the THEMIS probes when they were located in the magnetotail, within the region $-30 R_{\mathrm{E}}<X_{\mathrm{gsm}}<-6 R_{\mathrm{E}}$ and $\sqrt{Y_{\mathrm{gsm}}^{2}+Z_{\mathrm{gsm}}^{2}}<12 R_{\mathrm{E}}$ during the 2007 to 2011 tail seasons. The selection criterion used by Liu et al. (2013a) to define a DF is that there should be rapid $B_{z}$ increase in GSM coordinates $\left(\frac{\mathrm{d} B_{z}}{\mathrm{~d} t}>0.5 \mathrm{nT} \mathrm{s}^{-1}\right.$ applied to a three-point running average of the $3 \mathrm{~s}$ resolution fluxgate spin-fit (FGS) magnetic field data. The first point that meets this criterion is defined as the event's start $\left(t_{\mathrm{s}}\right)$ (see Liu et al. (2013a) for more details). In this study, we use the time corresponding to the minimum value of $B_{z}$ between $t_{\mathrm{s}}-10 \mathrm{~s}$ and $t_{\mathrm{s}}$ as our epoch zero reference $\left(t_{0}\right)$. We further select the events in which the average plasma $\beta$ value (the ratio of thermal pressure to magnetic pressure) prior to the event, between $t_{0}-4 \mathrm{~min}$ and $t_{0}-3 \mathrm{~min}$, is $>1$ in order to ensure the spacecraft was located in the central plasma sheet. This is a higher value of $\beta$ than adopted in early studies to identify the central plasma sheet (Angelopoulos et al., 1993; Baumjohann et al., 1989), although lower than the value suggested by Walsh et al. (2011) in a recent statistical study of particle distribution function in the magnetotail using Cluster data. We select $t_{0}-4 \mathrm{~min}$ to $t_{0}-3 \mathrm{~min}$ as the background to avoid contaminating this calculated average with any variations in the plasma $\beta$ immediately prior to the arrival of the DF. All results in this paper are presented in GSM coordinates.

\subsection{Statistical results}

Figure 1 shows the results of a superposed epoch analysis of magnetic field $B_{x}$ (left hand column) and $B_{z}$ (centre column) components and the ion thermal pressure (right hand column) in the interval $t_{0}-5 \mathrm{~min}$ to $t_{0}+5 \mathrm{~min}$. We perform this statistical analysis for varied distances from the nominal magnetotail neutral sheet (NS) using the strength of the background field, $\mathrm{B}_{x, 0}$ as a proxy for distance. The top row (panels, a, $\mathrm{f}$ 

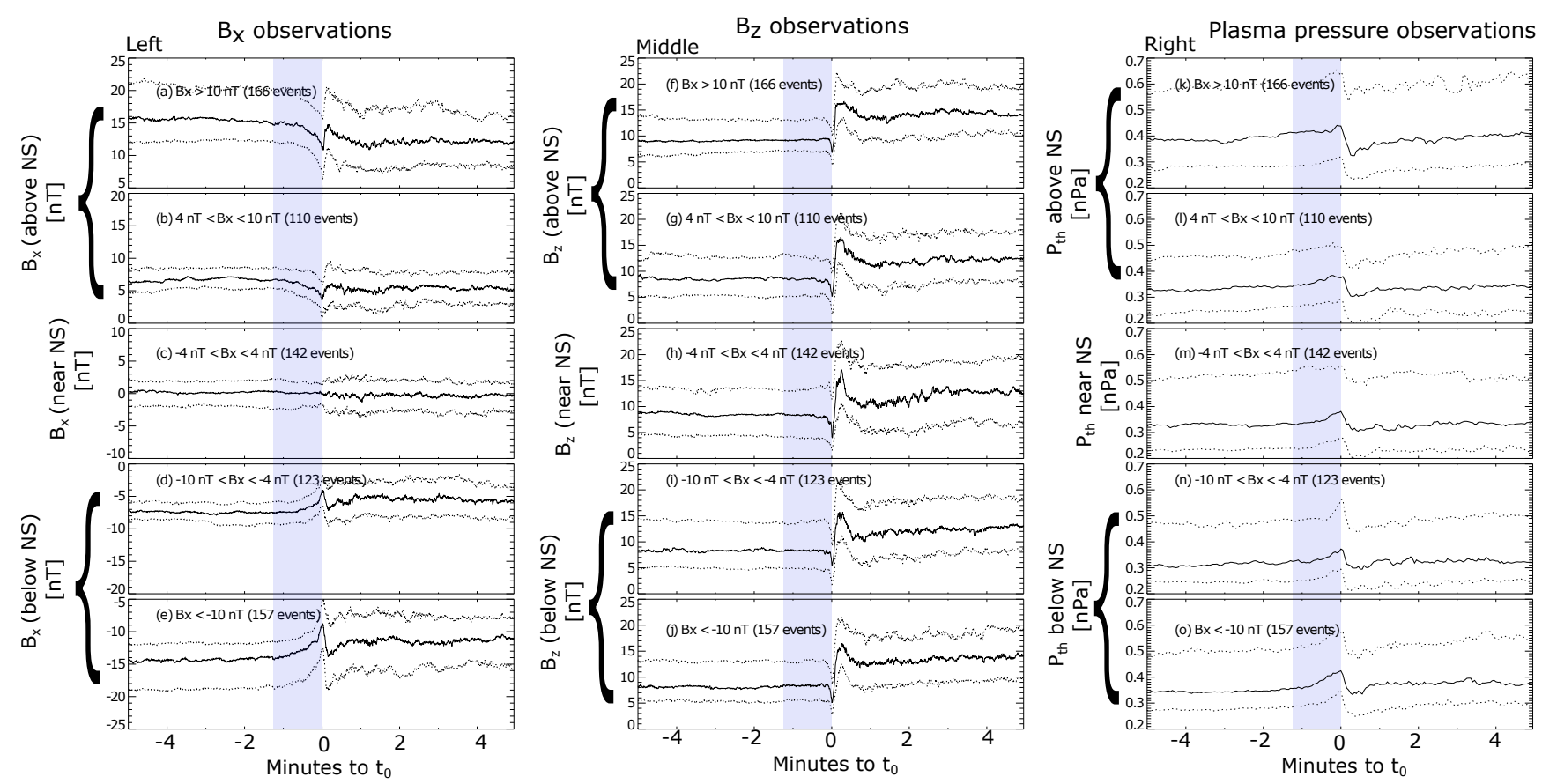

Figure 1. Superposed epoch analysis of the characteristics of DFs for $B_{x}$ (Left column), $B_{z}$ (Middle) and plasma pressure (Right). For each of the three columns, plotted from the top to bottom are the superposed results from DFs with background magnetic field $B_{x}>10 \mathrm{nT}$, $4 \mathrm{nT}<B_{x}<10 \mathrm{nT},-4 \mathrm{nT}<B_{x}<4 \mathrm{nT},-10 \mathrm{nT}<B_{x}<-4 \mathrm{nT}$ and $B_{x}<-10 \mathrm{nT}$. The three curves in each panel denote the upper quartile (dotted), median (solid), and lower quartile (dotted) of the superposed data.

and $\mathrm{k})$, shows data from well above the NS $\left(B_{x, 0}>10 \mathrm{nT}\right)$; the second row (panels $\mathrm{b}, \mathrm{g}$ and $\mathrm{l}$ ) shows data from above the NS $\left(4 \mathrm{nT}<B_{x, 0}<10 \mathrm{nT}\right)$; the middle row (c, $\mathrm{h}$ and $\left.\mathrm{m}\right)$ shows data near the NS $\left(-4 \mathrm{nT}<B_{x, 0}<4 \mathrm{nT}\right)$; the fourth row (d, i and n) shows data from below the NS $\left(-10 \mathrm{nT}<B_{x, 0}<-4 \mathrm{nT}\right)$ and the bottom row (e, $\mathrm{j}$ and o) shows data from well below the NS $\left(B_{x, 0}<-10 \mathrm{nT}\right)$. The number of individual events contributing to each superposed epoch analysis is shown in each panel. The three curves plotted in each panel are the upper quartile (dotted), median (solid), and lower quartile (dotted) of the superposed data. The plots in the left column show that at distances further from the neutral sheet in both hemispheres, $\left|B_{x}\right|$ decreases on average $\sim 1.5 \mathrm{~min}$ before the arrival of the DF (in the period shown by the shaded box), while the plasma pressure, shown in the right hand column increases over the same period. At this time, $B_{z}$ (middle column) remains near constant but shows a modest but rapid decrease about $6 \mathrm{~s}$ before the sharp increase representative of the DF itself. This $B_{z}$ signature is seen for all distance ranges shown in the figure. Yao et al. (2013) showed that such a dip in the magnetic field ahead of DFs is a common, and found that field-aligned currents usually exist in this region.

We now compare DFs based on the magnitude of the change in $B_{z}$ prior to the DF arrival. We create the value $B_{z \text {,dip }}$, as $\left|B_{z}\left(t_{0}\right)-B_{z, 0}\right|$. Here, $B_{z, 0}$ is calculated as the average of $B_{z}$ between $t_{0}-4 \mathrm{~min}$ to $t_{0}-3 \mathrm{~min}$. Figure 2 shows the results of a superposed epoch analysis of magnetic field $B_{x}$,
$B_{z}$ and plasma thermal pressure for DFs with small $B_{z \text {,dip }}$ ( $<4 \mathrm{nT}$, panels a, c and e) or large $B_{z, \text { dip }}(>4 \mathrm{nT}$, panels b, d and f). We reverse the sign of $B_{x}$ for events with $B_{x, 0}<0$ to study the overall trends of the evolution of $B_{x}$ through DFs. Figure $2 \mathrm{~g}$ shows the plasma thermal pressure detrended by the average pressure between $t_{0}-4 \mathrm{~min}$ and $t_{0}-3 \mathrm{~min}$ for both these groups of DFs. Again it is clear that the plasma pressure increases from about $1.5 \mathrm{~min}$ before the arrival of the DF. This plasma pressure increase is accompanied by a magnetic field $B_{x}$ decrease, while the $B_{z}$ remains approximately constant until several seconds prior to the arrival of the DF. Figure $2 \mathrm{~g}$ also shows that the pressure build-up ahead of DF for larger $B_{z}$ dip events $(\delta p \sim 0.08 \mathrm{nPa})$ is greater than small $B_{z}$ dip events $(\delta p \sim 0.04 \mathrm{nPa})$, but occurs over the same length of time. The relationship between the size of pressure change

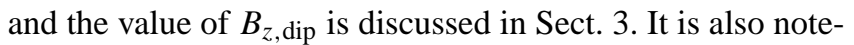
worthy, comparing Fig. 2 (panels c and d), that the magnetic field $B_{x}$ in the undisturbed region before the DF's arrival is similar in each case, but decreases more significantly for the large $B_{z \text {,dip }}$ group. Since the plasma pressure in the undisturbed region is different for the large and small $B_{z \text {,dip }}$ groups (comparing panels e and f), we suggest that this difference is due to different magnetotail conditions, such as the different strength of the cross-tail current or level of geomagnetic activity, rather than different average distances from the neutral sheet. However, this needs a further study. 


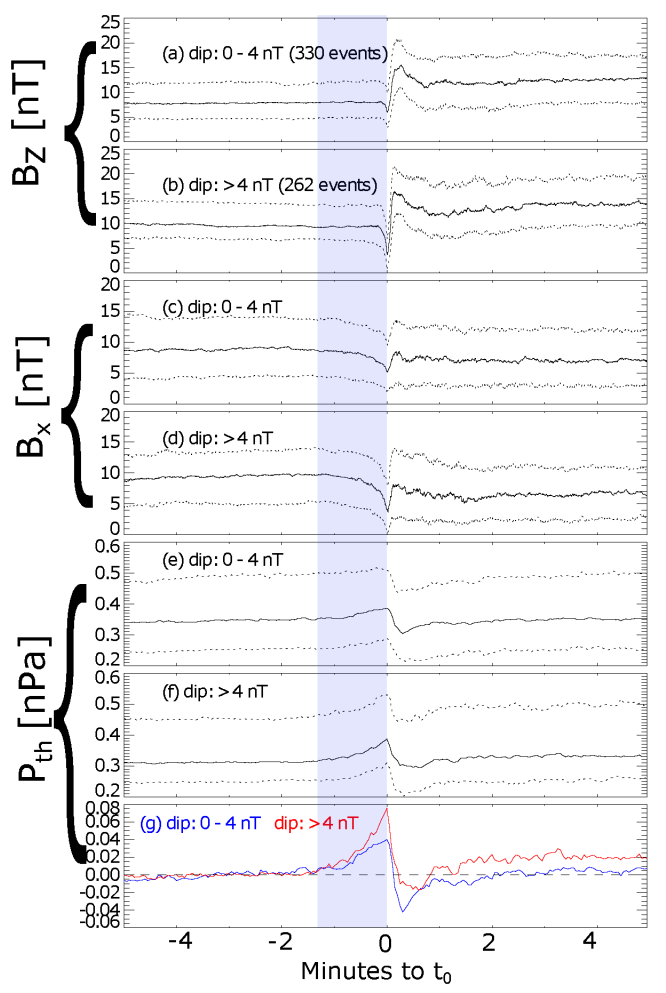

Figure 2. Superposed epoch analysis of (a and b) magnetic field $B_{z}$ for both DF with small dip ( $B_{z}$,dip from 0 to $4 \mathrm{nT}$ ) and large dip ( $B_{z, \text { dip }}>4 \mathrm{nT}$ ); (c and d) magnetic field $B_{x}$ for both DF groups. We reversed the sign of $B_{x}$ for events with $B_{x, 0}<0$; (e and f) plasma pressure for both DF groups and (g) plasma pressure for both DF groups subtracted with their average values of the time range from $t_{0}-4 \mathrm{~min}$ to $t_{0}-3 \mathrm{~min}$. The three curves in each panel denote the upper quartile (dotted), median (solid), and lower quartile (dotted) of the superposed data.

\section{The re-distribution of cross-tail current density}

By assuming that the magnetic field in the pressure buildup region before the arrival of DF can be represented by the combination of a magnetic dipole and a one-dimensional current sheet, as suggested in Lui (2011), we can derive the current evolution as the DF approaches. Specifically, we assume the observed magnetic field profile is given by $\boldsymbol{B}_{\text {obs }}(x, y, z)=\boldsymbol{B}_{\text {dipole }}(x, y, z)+\boldsymbol{B}_{\mathrm{cs}}(z)$, where $\boldsymbol{B}_{\text {dipole }}(x, y, z)$ is the dipole magnetic field, and $\boldsymbol{B}_{\mathrm{cs}}(z)$ is the magnetic field contributed by the current sheet. This latter field is in $X$-direction for a one-dimensional cross-tail current sheet which lies in the $X Y$ plane with current flowing purely in the $Y$ direction. The current density is then given by Ampère's law:

$\mu_{0} \boldsymbol{J}=\nabla \times \boldsymbol{B}=\nabla \times\left(\boldsymbol{B}_{\text {dipole }}+\boldsymbol{B}_{\mathrm{cs}}\right)=\nabla \times \boldsymbol{B}_{\mathrm{cs}}$.

Note that we do not consider the displacement current in Eq. (1). The zero current density from dipole magnetic field integration is applied in Eq. (1). Under our assumptions, changes of the cross-tail current can thus be expressed as

$\delta J_{y}=\frac{1}{\mu_{0}} \frac{\partial\left(\delta B_{x, \mathrm{cs}}\right)}{\partial z}$

Considering that $B_{x, \mathrm{obs}}=B_{x, \text { dipole }}+B_{x, \mathrm{cs}}$ and $B_{x, \text { dipole does }}$ not significant change over the few minutes of an observation, then we are able to obtain a relationship between the changes of the observed magnetic field $B_{x}$ and the cross-tail current:

$\delta J_{y}=\frac{1}{\mu_{0}} \frac{\partial\left(\delta B_{x, \mathrm{obs}}\right)}{\partial z}$

In Eqs. (2) and (3), we ignore the variation in the $Z$ component of the magnetic field with $X$ position since, as shown in middle column of Fig. 1, the magnetic field $B_{z}$ does not significantly change in the pressure build-up region (shaded) until several seconds before the arrival of the DF. Thus our assumption is consistent with the observations presented within this paper.

Changes in $B_{x}$ observed by a single spacecraft may be the result of current sheet flapping (Runov et al., 2009). However, the changes of $B_{x}$ and plasma pressure ahead of DFs shown in Fig. 1 are not a consequence of plasma sheet flapping, since $\left|B_{x}\right|$ decreases (plasma pressure increases) both above and below the neutral sheet. We suggest that these characteristics are a result of the compression of plasma ahead of the DF. Liu et al. (2013a) also showed that $\left|B_{x}\right|$ tends to decrease before the arrival of DF, and explained this $\left|B_{x}\right|$ decrease as a consequence of duskward currents on a DF structure which is concave when viewed in the $X Z$ plane. The left hand column of Fig. 1 shows that the change in the $X$-component of the magnetic field is larger in the outer plasma sheet (panels a and e) than nearer the centre (panels $\mathrm{b}$ and $\mathrm{d}$ ). Based on the observations that the $\left|B_{x}\right|$ decrease is in concert with the pressure increase over several minutes, while the $B_{Z}$ decrease only occurs within $\sim 6 \mathrm{~s}$ of the DF's arrival in the central plasma sheet region, we suggest that a small-scale duskward current on the DF itself is only able to significantly affect the limited region passing the spacecraft over the few seconds immediately prior to the DF's arrival, while the longer duration of the $\left|B_{x}\right|$ decrease over the blue shaded region must occur mainly as a result of a redistribution in the larger-scale cross-tail current ahead of the DF.

We obtain the $\delta B_{x, \text { obs }}$ for the calculation in Eq. (3) by subtracting the mean between $t_{0}-4 \mathrm{~min}$ to $t_{0}-3 \mathrm{~min}$ of the statistical median of $B_{x, \text { obs. }}$ To estimate the total current redistribution in the pressure build-up region, we use the $\delta B_{x}$,obs from the DFs with $B_{x, 0}>10 \mathrm{nT}$ (Fig. 1a) and $B_{x, 0}<-10 \mathrm{nT}$ (Fig. 1e). The median of the statistical $\left|B_{x, 0}\right|$ in both hemispheres is about $15 \mathrm{nT}$. Since the current density in the current sheet is usually a few $\mathrm{nA} \mathrm{m}^{-2}$ (Mitchell et al., 1990), the corresponding $\mathrm{d} B_{x} / \mathrm{d} z$ should be a few $\mathrm{nT} / 1000 \mathrm{~km}$. Runov et al. (2004) and Yao et al. (2014b) have also shown that $\mathrm{d} B_{x} / \mathrm{d} z$ is $2-4 \mathrm{nT} / 1000 \mathrm{~km}$ from multi-spacecraft observations. We thus assume that observations in the two 
groups are separated by $1 R_{\mathrm{E}}$ across the neutral sheet, we are then able estimate an average redistributed current density before the arrival of DF. The blue and black curves in Fig. 3a show the superposed epoch analysis results of $B_{x \text {,obs }}$ for the DF events observed above $\left(B_{x, 0}>10 \mathrm{nT}\right)$ and below $\left(B_{x, 0}<-10 \mathrm{nT}\right)$ the magnetotail neutral sheet respectively. The red curve in Fig. 3a presents the detrended difference of $\delta B_{x}$,obs between the two hemispheres, i.e. $\delta B_{x \text {, obs }}$ (tot $)=$ $\delta B_{x, \text { obs }}$ (north) $-\delta B_{x, \text { obs }}$ (south).

We can obtain the total change in current by integrating along $X$ - and $Z$-directions.

$$
\begin{aligned}
\Delta J_{y} & =\iint \frac{1}{\mu_{0}} \frac{\partial\left(\delta B_{x}(x)\right)}{\partial z} \mathrm{~d} x \mathrm{~d} z=\frac{1}{\mu_{0}} \int_{x_{\mathrm{UP}}}^{x_{\mathrm{DF}}} \delta B_{x}(x) \mathrm{d} x \\
& =\frac{1}{\mu_{0}} \int_{t_{\mathrm{UP}}}^{t_{\mathrm{DF}}} \delta B_{x}(t) \mathrm{d}\left(v_{\mathrm{DF}} t\right) .
\end{aligned}
$$

In Eq. (4), the integration in $x$ is over the distance to the DF in the compression region, which can be expressed as a function of time by assuming that this region is propagating at the same (time-steady) speed as the DF's speed $\left(v_{\mathrm{DF}}\right)$. We take this speed $\sim 120 \mathrm{~km} \mathrm{~s}^{-1}$ based on the superposed epoch analyses of the $X$-component of the perpendicular bulk velocity shown in Fig. $3 b$ and c. We note that the relation between the propagation speed of a DF and the bulk velocity of the associated plasma is not well understood yet. However, for the purposes of this study, our main conclusion is not critically dependent on a precise DF propagation velocity and hence we approximate the DF propagation velocity as the observed bulk plasma velocity. The subscripts "UP" and "DF" in Eq. (4) represent un-perturbed region and the start of dipolarization front. For the limits of the integration we use $t_{\mathrm{UP}}=t_{0}-1.5 \mathrm{~min}$ and $t_{\mathrm{DF}}=t_{0}$. The decrease of current in the $Y$-direction, $\Delta J_{y}$ is calculated to be $2.5 \times 10^{4} \mathrm{~A}$. This value is more than one order of magnitude smaller than the cross-tail current reduction that occurs in a typical substorm, i.e. $10^{6}$ A (McPherron et al., 1973). We thus believe this current redistribution may not be very important in a large-scale substorm current system.

The plasma pressure increases over the period of $\sim 1.5 \mathrm{~min}$ before the DF arrives, which suggests a pressure gradient towards the DF. The equivalent dawnward diamagnetic current associated with the pressure gradient towards the DF can be estimated in MHD theory. The acceleration/deceleration of a plasma flow is usually considered as a whole structure, which thus corresponds to a uniform current density around the DF. The uniform current density is not consistent with the magnetic dip feature. While the pressure gradient shows bidirectional feature that is consistent with the magnetic field variations. We thus mainly discuss the diamagnetic current associated with the pressure gradient in this paper. Using the plasma and magnetic field parameters from the DFs near the central plasma sheet (Fig. 1c, h and $\mathrm{m}$ ) to estimate a current

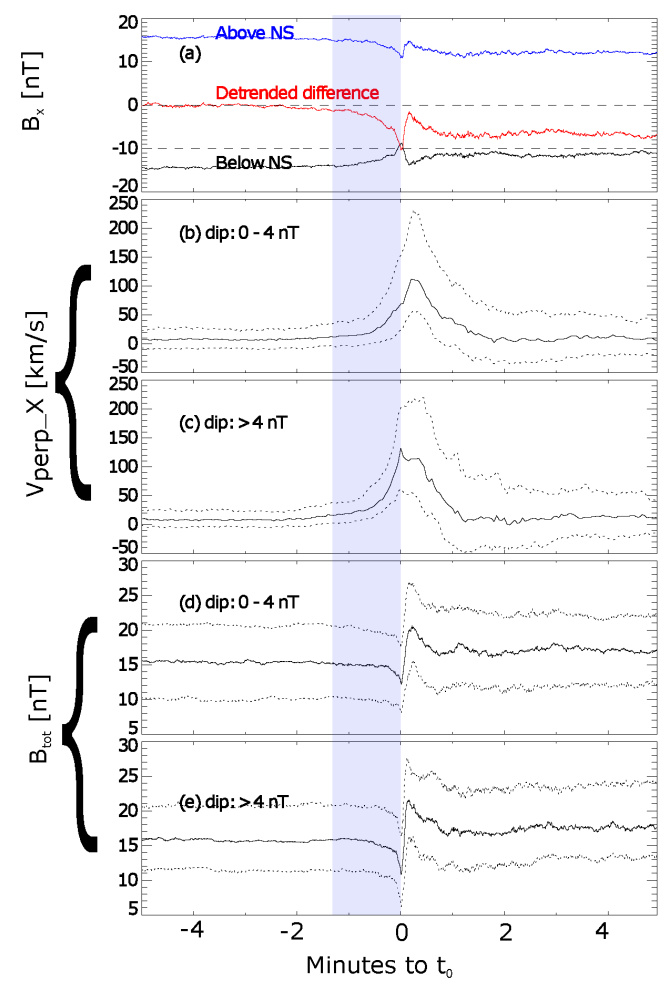

Figure 3. (a) The black curves show the superposed epoch analysis of magnetic field $B_{x}$ for DF events at both hemispheres; the red curve shows the difference in $\delta B$ between both hemispheres, which were obtained by subtracting the average value between $t_{0}-4 \mathrm{~min}$ to $t_{0}-3$ min from the statistical median. (b, c) The $X$-component of perpendicular bulk velocity for both DF groups.

density change in the pressure build-up region.

$\Delta J_{y}=\left(\frac{\boldsymbol{B} \times \nabla P}{B^{2}}\right)_{y}$

Figure 1 shows that $B_{z}$ at the NS is about $9 \mathrm{nT}$ until $\sim 6 \mathrm{~s}$ before the DF's arrival, and the pressure increases by 0.05 $\mathrm{nPa}$ over about $1.5 \mathrm{~min}$. The value is also similar from the lower and upper quartiles as shown in Fig. 2e and 2f. Assuming that the DF propagates with $\sim 120 \mathrm{~km} / \mathrm{s}$, we estimate a spatial scale of the pressure build up region to be $\sim 1.7$ $R_{\mathrm{E}}$, thus the equivalent outward pressure gradient is roughly $\sim 0.029 \mathrm{nPa} / R_{\mathrm{E}}$. From Eq. (5), we obtain a dawnward current density of $\sim 0.5 \mathrm{nA} \mathrm{m}^{-2}$ associated with the pressure build-up. The total current redistribution is then $\sim 3.4 \times 10^{4}$ A, which is comparable to the current redistribution derived from the $\left|B_{x}\right|$ decrease $\left(2.5 \times 10^{4} \mathrm{~A}\right)$. The small difference may be a result of uncertainties in the thickness of the current sheet, or due to our estimate of the current density using observations at the NS, which may provide a higher value than the average over the full current sheet, or our simplification of a nonlinear plasma pressure gradient. Future observations from multi-probes with small spatial separations and high temporal resolution particle observations, such as Mag- 
netospheric Multiscale (MMS) Mission (Mauk et al., 2014) facilitate improved estimates of these variables.

Finally, we note that for a dipolarization process, the $B_{z}$ increase should be accompanied by $B_{x}$ decrease across the event (Lui, 1996; Takahashi et al., 1987). However, the left hand columns of Fig. 1 show that the $\left|B_{x}\right|$ sharply increases across the DF in tandem with the increase in $B_{z}$, which suggest the dipolarization fronts identified in this study are strong magnetic flux boundaries, but are not consistent with the dipolarization process which develops as part of the large-scale substorm current wedge, which is also accompanied by a strong magnetic perturbation and usually lasts for several minutes to tens of minutes. The two types of dipolarization have been discussed by Nakamura et al. (2009) and Lui (2014).

The change of current density associated with the pressure buildup may not result in any field-aligned currents connecting the magnetosphere and ionosphere. The volume gradient of the flux tube with one unit magnetic flux is essential to evaluate the field-aligned current associated with the plasma pressure gradient (Vasyliunas, 1970). However, the volume gradient of magnetic flux tube is extremely difficult to be accurately estimated in any magnetic field model in the perturbed magnetotail, especially accompanied with BBFs (Kubyshkina et al., 2011; Tsyganenko, 1995). Moreover, Liemohn et al. (2013) suggested that current could flow in a closed loop around peaks in plasma pressure forming a banana current. This banana current is distinct from the other current systems in the near-Earth space. It is thus possible that the current density change ahead of DF may be a certain part of a current loop in a banana current related to the pressure peak, as opposed to closing to the ionosphere via FACs.

To understand how the current in the dip region circuit with the magnetotail current system, we compare the magnetic and plasma features around DFs with big magnetic dip and small dip. Figure 4a gives the detrended differences of magnetic field $B_{z}$ and plasma pressure for the two groups of DFs in Fig. 2. From the magnetic differences, we clearly see that there are three trends $\left(B_{z}\right.$ decrease, increase and decrease) from $t_{0}-10 \mathrm{~s}$ to $t_{0}+10 \mathrm{~s}$, as indicated by the shaded region. From Ampère's Law, the magnetic variations imply three current structures in this region; a dawnward current, duskward current and a final dawnward current. The pressure differences also show three trends correlated with the $B_{z}$ trend. The peak plasma pressure exists in the magnetic dip region, which suggests that the current system is a banana current loop. Figure $4 \mathrm{~b}$ is a cartoon illustrating the banana current loop between the magnetic dip region and the dipolarization front layer. This cartoon presents similar current system as the current system associated with DF in Yao et al. (2013), however, they represent a very different physical process. In Yao et al. (2013), the dawnward and duskward currents are the current system of DF, while here the dawnward and duskward currents are additional structures asso- (a)

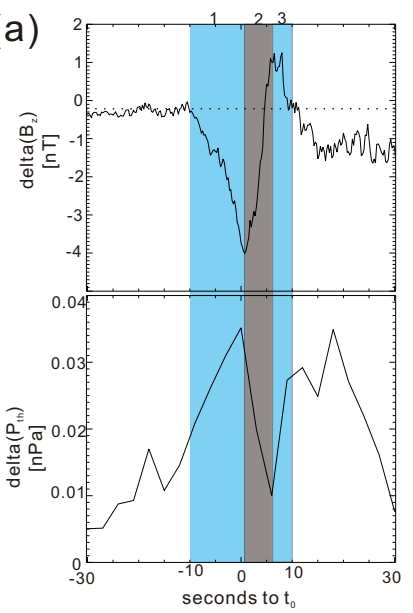

(b)

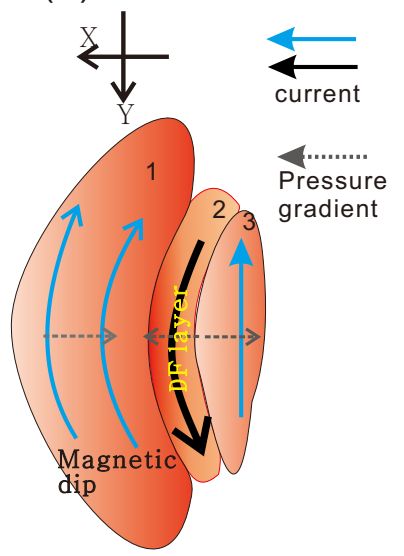

Figure 4. Illustration of the current system associated with the magnetic dip ahead of DF. (a) The difference of magnetic field $B_{z}$ and plasma pressure between big dip events and small dip events. The variations were obtained by subtracting the average value between $t_{0}-4$ min to $t_{0}-3$ min from the statistical median. (b) A cartoon to show the current system of magnetic $B_{z}$ dip, derived from the different variations of magnetic field $B_{z}$ and plasma pressure between big dip events and small dip events.

ciated with the magnetic dip. In addition, the difference of magnetic field $B_{z}$ is almost the same at $t_{0}-10 \mathrm{~s}$ and $t_{0}+10 \mathrm{~s}$, which strongly suggests that these three current form circuits, which are independent from the background magnetotail current. We point out that although the plasma pressure variation supports the picture of banana current loop, it may be not exactly the same as described in Liemohn et al. (2013) since the DF is a kinetic structure that can not be fully interpreted by MHD theory. For the third current structure in Fig. 4 that was not shown in the current system described in Fig. 4b by Yao et al. (2013), we suggest this pressure increase may be caused by the compression of the plasma flow behind the DF. This dawnward current is naturally expected from Fig. $2 \mathrm{~g}$, in which the plasma pressure suddenly decreases on the DF layer and thereafter returns to a slightly higher value. This slight recovery in plasma pressure corresponds to a dawnward current as shown in Fig. 4. However how the flow compresses this region requires further study in the future. Fig. 4 only presents the current close to the DF, rather than the current system of a whole DFB, which may carry significant FACs and play an important role in magnetosphere-ionosphere coupling (Birn and Hesse, 2013; Birn et al., 2004; Yao et al., 2012).

It is interesting to note that the peak velocity of the flow appear in different places relative to the DF for the small dip events and large dip events, as shown in Fig. 3. The DF exist on the leading edge of the flow for the small dip group, while exist on the peak of the flow for the big dip group. Hamrin et al. (2014) suggested that relatively different places represent an evolution of lifecycle. Our results also show that the dip 
is generated by the interaction between DF and the ambient plasma, which is consistent with their conclusion.

\section{Summary}

Using a database of 698 DF events observed by THEMIS in plasma sheet, we have shown that in the $\sim 1.5 \mathrm{~min}$ ahead of the arrival of a dipolarization front, there is an increase in the thermal plasma pressure and a corresponding decrease in the absolute value of the $X$-component of the magnetic field, $\left|B_{x}\right|$. We have shown that the total current change from the variation in $B_{x}$ is comparable to that associated with the pressure gradient, indicating that the reduction in the cross tail current ahead of a DF is due to the compression of plasma ahead of the flow. In addition, the total current change is an order smaller than the typical total current associated with a moderate substorm current wedge. The redistribution of cross-tail current in the region ahead of the DF, combining with the duskward current on the DF could produce the dip in $B_{z}$ component immediately ahead of the DF. We also showed that larger dips in $B_{z}$ are associated with a larger change in plasma pressure ahead of a DF.

Acknowledgements. This work is supported by UK Science and Technology Facilities Council (STFC) grant (ST/L005638/1) at UCL/MSSL. IJR is supported by STFC grant (ST/L000563/1) and National Environmental Research Council (NERC) grant (NE/L007495/1 and NE/M00886X/1) and CF is supported by NERC grant (NE/L007495/1 and NE/M00886X/1). Jiang Liu is supported by NASA contract NAS5-02099 and grant NNX14AC17G. Zhonghua Yao thanks Tony Lui, Andrew Fazakerley, Ali Varsani and Dimitry Pokhotelov for helpful discussions. We acknowledge NASA contract NAS5-02099 and V. Angelopoulos for use of data from the THEMIS Mission. Specifically: D. Larson and R. P. Lin for use of SST data; C. W. Carlson and J. P. McFadden for use of ESA data and K. H. Glassmeier, U. Auster and W. Baumjohann for the use of FGM data provided under the lead of the Technical University of Braunschweig and with financial support through the German Ministry for Economy and Technology and the German Center for Aviation and Space (DLR) under contract 50 OC 0302. All THEMIS data can be obtained from http://themis.ssl.berkeley.edu/data/themis/.

The topical editor L. Blomberg thanks A. Lui and one anonymous referee for help in evaluating this paper.

\section{References}

Angelopoulos, V.: The THEMIS mission, Space Sci. Rev., 141, 534, 2008.

Angelopoulos, V., Baumjohann, W., Kennel, C. F., Coroniti, F. V., Kivelson, M. G., Pellat, R., Walker, R. J., Luhr, H., and Paschmann, G.: Bursty Bulk Flows in the Inner Central Plasma Sheet, J. Geophys. Res.-Space, 97, 4027-4039, 1992.

Angelopoulos, V., Kennel, C. F., Coroniti, F. V., Pellat, R., Spence, H. E., Kivelson, M. G., Walker, R. J., Baumjohann, W., Feldman,
W. C., Gosling, J. T., and Russell, C. T.: Characteristics of ion flow in the quiet state of the inner plasma sheet, Geophys. Res. Lett., 20, 1711-1714, doi:10.1029/93GL00847, 1993.

Angelopoulos, V., Kennel, C., Coroniti, F., Pellat, R., Kivelson, M., Walker, R., Russell, C., Baumjohann, W., Feldman, W., and Gosling, J.: Statistical Characteristics of Bursty Bulk Flow Events, J. Geophys. Res., 99, 21257-21280, 1994.

Angelopoulos, V., Runov, A., Zhou, X.-Z., Turner, D. L., Kiehas, S. A., Li, S.-S., and Shinohara, I.: Electromagnetic Energy Conversion at Reconnection Fronts, Science, 341, 1478-1482, 2013.

Auster, H. U., Glassmeier, K. H., Magnes, W., Aydogar, O., Baumjohann, W., Constantinescu, D., Fischer, D., Fornacon, K. H., Georgescu, E., Harvey, P., Hillenmaier, O., Kroth, R., Ludlam, M., Narita, Y., Nakamura, R., Okrafka, K., Plaschke, F., Richter, I., Schwarzl, H., Stoll, B., Valavanoglou, A., and Wiedemann, M.: The THEMIS Fluxgate Magnetometer, Space Sci. Rev., 141, 235-264, 2008.

Baumjohann, W., Paschmann, G., and Cattell, C. A.: Average Plasma Properties in the Central Plasma Sheet, J. Geophys. Res.Space, 94, 6597-6606, 1989.

Birn, J. and Hesse, M.: Details of current disruption and diversion in simulations of magnetotail dynamics, J. Geophys. Res.-Space, 101, 15345-15358, 1996.

Birn, J. and Hesse, M.: The substorm current wedge in MHD simulations, J. Geophys. Res.-Space, 118, 3364-3376, 2013.

Birn, J., Hesse, M., Haerendel, G., Baumjohann, W., and Shiokawa, K.: Flow braking and the substorm current wedge, J. Geophys. Res.-Space, 104, 19895-19903, 1999.

Birn, J., Raeder, J., Wang, Y. L., Wolf, R. A., and Hesse, M.: On the propagation of bubbles in the geomagnetic tail, Ann. Geophys., 22, 1773-1786, doi:10.5194/angeo-22-1773-2004, 2004.

Forsyth, C., Lester, M., Cowley, S. W. H., Dandouras, I., Fazakerley, A. N., Fear, R. C., Frey, H. U., Grocott, A., Kadokura, A., Lucek, E., Rème, H., Milan, S. E., and Watermann, J.: Observed tail current systems associated with bursty bulk flows and auroral streamers during a period of multiple substorms, Ann. Geophys., 26, 167-184, doi:10.5194/angeo-26-167-2008, 2008.

Forsyth, C., Fazakerley, A. N., Rae, I. J., Watt, C., Murphy, K., Wild, J. A., Karlsson, T., Mutel, R., Owen, C. J., and Ergun, R.: In situ spatiotemporal measurements of the detailed azimuthal substructure of the substorm current wedge, J. Geophys. Res.-Space, 119, 927-946, doi:10.1002/2013JA019302, 2014.

Fu, H. S., Khotyaintsev, Y. V., Andre, M., and Vaivads, A.: Fermi and betatron acceleration of suprathermal electrons behind dipolarization fronts, Geophys. Res. Lett., 38, L16104, doi:10.1029/2011GL048528, 2011.

Fu, H. S., Cao, J. B., Khotyaintsev, Y. V., Sitnov, M. I., Runov, A., Fu, S. Y., Hamrin, M., Andre, M., Retino, A., Ma, Y. D., Lu, H. Y., Wei, X. H., and Huang, S. Y.: Dipolarization fronts as a consequence of transient reconnection: In situ evidence, Geophys. Res. Lett., 40, 6023-6027, 2013.

Hamrin, M., Pitkanen, T., Norqvist, P., Karlsson, T., Nilsson, H., Andre, M., Buchert, S., Vaivads, A., Marghitu, O., Klecker, B., Kistler, L. M., and Dandouras, I.: Evidence for the braking of flow bursts as they propagate toward the Earth, J. Geophys. Res.Space, 119, 9004-9018, 2014.

Kronberg, E. A., Woch, J., Krupp, N., Lagg, A., Khurana, K. K., and Glassmeier, K. H.: Mass release at Jupiter: Substorm-like pro- 
cesses in the Jovian magnetotail, J. Geophys. Res., 110, A03211, doi:10.1029/2004JA010777, 2005.

Kubyshkina, M., Sergeev, V., Tsyganenko, N., Angelopoulos, V., Runov, A., Donovan, E., Singer, H., Auster, U., and Baumjohann, W.: Time-dependent magnetospheric configuration and breakup mapping during a substorm, J. Geophys. Res., 116, A00I27, doi:10.1029/2010JA015882, 2011.

Liemohn, M. W., Ganushkina, N. Y., Katus, R. M., De Zeeuw, D. L., and Welling, D. T.: The magnetospheric banana current, J. Geophys. Res.-Space, 118, 1009-1021, 2013.

Lui, A.: Reduction of the cross-tail current during near-Earth dipolarization with multisatellite observations, J. Geophys. Res., 116, A12239, doi:10.1029/2011JA017107, 2011.

Lui, A. T. Y.: Current disruption in the Earth's magnetosphere: Observations and models, J. Geophys. Res.-Space, 101, 1306713088, 1996.

Lui, A. T. Y.: Evidence for two types of dipolarization in the earth's magnetotail, J. Atmos. Sol.-Terr. Phy., 115, 17-24, 2014.

Liu, J., Angelopoulos, V., Runov, A., and Zhou, X. Z.: On the current sheets surrounding dipolarizing flux bundles in the magnetotail: The case for wedgelets, J. Geophys. Res.-Space, 118, 20002020, 2013a.

Liu, J., Angelopoulos, V., Zhou, X. Z., Runov, A., and Yao, Z. H.: On the role of pressure and flow perturbations around dipolarizing flux bundles, J. Geophys. Res.-Space, 118, 7104-7118, $2013 b$.

Liu, J., Angelopoulos, V., Zhou, X. Z., and Runov, A.: Magnetic flux transport by dipolarizing flux bundles, J. Geophys. Res.Space, 119, 909-926, 2014.

Liu, J., Angelopoulos, V., Chu, X. N., Zhou, X. Z., and Yue, C.: Substorm current wedge composition by wedgelets, Geophys. Res. Lett., 42, 1669-1676, 2015.

Mauk, B., Blake, J., Baker, D., Clemmons, J., Reeves, G., Spence, H., Jaskulek, S., Schlemm, C., Brown, L., and Cooper, S.: The energetic particle detector (EPD) investigation and the energetic ion spectrometer (EIS) for the magnetospheric multiscale (MMS) mission, Space Sci. Rev., 2014, 1-44, 2014.

McFadden, J. P., Carlson, C. W., Larson, D., Ludlam, M., Abiad, R., Elliott, B., Turin, P., Marckwordt, M., and Angelopoulos, V.: The THEMIS ESA Plasma Instrument and In-flight Calibration, Space Sci. Rev., 141, 277-302, 2008.

McPherron, R., Russell, C., and Aubry, M.: 9. Phenomenological Model for Substorms, J. Geophys. Res., 78, 3131-3149, 1973.

Mitchell, D. G., Williams, D. J., Huang, C. Y., Frank, L. A., and Russell, C. T.: Current carriers in the near-Earth cross-tail current sheet during substorm growth phase, Geophys. Res. Lett., 17, 583-586, 1990.

Nakamura, R., Baumjohann, W., Klecker, B., Bogdanova, Y., Balogh, A., Reme, H., Bosqued, J. M., Dandouras, I., Sauvaud, J. A., Glassmeier, K. H., Kistler, L., Mouikis, C., Zhang, T. L., Eichelberger, H., and Runov, A.: Motion of the dipolarization front during a flow burst event observed by Cluster, Geophys. Res. Lett., 29, 1942, doi:10.1029/2002GL015763, 2002.

Nakamura, R., Retinò, A., Baumjohann, W., Volwerk, M., Erkaev, N., Klecker, B., Lucek, E. A., Dandouras, I., André, M., and Khotyaintsev, Y.: Evolution of dipolarization in the nearEarth current sheet induced by Earthward rapid flux transport, Ann. Geophys., 27, 1743-1754, doi:10.5194/angeo-27-17432009, 2009.
Ohtani, S., Shay, M. A., and Mukai, T.: Temporal structure of the fast convective flow in the plasma sheet: Comparison between observations and two-fluid simulations, J. Geophys. Res., 109, A03210, doi:10.1029/2003JA010002, 2004.

Pan, D.-X., Zhou, X.-Z., Shi, Q.-Q., Liu, J., Angelopoulos, V., Runov, A., Zong, Q.-G., and Fu, S.-Y.: On the generation of magnetic dips ahead of advancing dipolarization fronts, Geophys. Res. Lett., 42, 4256-4262, doi:10.1002/2015GL064369, 2015.

Rostoker, G.: Some Observational Constraints for Substorm Models, Geoph Monog Series, 64, 61-72, 1991.

Runov, A., Sergeev, V., Nakamura, R., Baumjohann, W., Vörös, Z., Volwerk, M., Asano, Y., Klecker, B., Rème, H., and Balogh, A.: Properties of a bifurcated current sheet observed on $29 \mathrm{Au}-$ gust 2001, Ann. Geophys., 22, 2535-2540, doi:10.5194/angeo22-2535-2004, 2004.

Runov, A., Angelopoulos, V., Sergeev, V. A., Glassmeier, K.-H., Auster, U., McFadden, J., Larson, D., and Mann, I.: Global properties of magnetotail current sheet flapping: THEMIS perspectives, Ann. Geophys., 27, 319-328, doi:10.5194/angeo-27-3192009, 2009.

Sergeev, V. A., Angelopoulos, V., Gosling, J. T., Cattell, C. A., and Russell, C. T.: Detection of localized, plasma-depleted flux tubes or bubbles in the midtail plasma sheet, J. Geophys. Res., 101, 10817-10810, 1996.

Sergeev, V., Angelopoulos, V., Apatenkov, S., Bonnell, J., Ergun, R., Nakamura, R., McFadden, J., Larson, D., and Runov, A.: Kinetic structure of the sharp injection/dipolarization front in the flow-braking region, Geophys. Res. Lett., Geophys. Res. Lett., 36, L21105, doi:10.1029/2009GL040658, 2009.

Sitnov, M. I., Buzulukova, N., Swisdak, M., Merkin, V. G., and Moore, T. E.: Spontaneous formation of dipolarization fronts and reconnection onset in the magnetotail, Geophys. Res. Lett., 40, 22-27, doi:10.1029/2012GL054701, 2013.

Slavin, J. A., Lepping, R. P., Gjerloev, J., Fairfield, D. H., Hesse, M., Owen, C. J., Moldwin, M. B., Nagai, T., Ieda, A., and Mukai, T.: Geotail observations of magnetic flux ropes in the plasma sheet, J. Geophys. Res.-Space, J. Geophys. Res., 108, 1015, doi:10.1029/2002JA009557, 2003.

Sun, W.-J., Slavin, J. A., Fu, S., Raines, J. M., Zong, Q.-G., Imber, S. M., Shi, Q., Yao, Z., Poh, G., Gershman, D. J., Pu, Z., Sundberg, T., Anderson, B. J., Korth, H., and Baker, D. N.: MESSENGER observations of magnetospheric substorm activity in Mercury's near magnetotail, Geophys. Res. Lett., 42, 3692-3699, doi:10.1002/2015GL064052, 2015.

Sundberg, T., Slavin, J. A., Boardsen, S. A., Anderson, B. J., Korth, H., Ho, G. C., Schriver, D., Uritsky, V. M., Zurbuchen, T. H., Raines, J. M., Baker, D. N., Krimigis, S. M., McNutt, R. L., and Solomon, S. C.: MESSENGER observations of dipolarization events in Mercury's magnetotail, J. Geophys. Res.-Space, 117, A00M03, doi:10.1029/2012JA017756, 2012.

Takahashi, K., Zanetti, L. J., Lopez, R. E., Mcentire, R. W., Potemra, T. A., and Yumoto, K.: Disruption of the Magnetotail Current Sheet Observed by Ampte/Cce, Geophys. Res. Lett., 14, 1019-1022, 1987.

Tang, C. L., Lu, L., Zhou, M., and Yao, Z. H.: THEMIS observations of electron acceleration associated with the evolution of substorm dipolarization in the near-Earth tail, J. Geophys. Res.Space, 118, 4237-4247, doi:10.1002/jgra.50418, 2013. 
Tsyganenko, N. A.: Modeling the Earth's magnetospheric magnetic field confined within a realistic magnetopause, J. Geophys. Res., 100, 5599-5612, 1995.

Vasyliunas, V. M.: Mathematical models of magnetospheric convection and its coupling to the ionosphere, in: Particles and Fields in the Magnetosphere, edited by: McCormac, B. M., D. Reidel, Hingham, MA, 60-71, 1970.

Vogiatzis, I. I., Isavnin, A., Zong, Q.-G., Sarris, E. T., Lu, S. W., and Tian, A. M.: Dipolarization fronts in the near-Earth space and substorm dynamics, Ann. Geophys., 33, 63-74, doi:10.5194/angeo-33-63-2015, 2015.

Walsh, A. P., Owen, C. J., Fazakerley, A. N., Forsyth, C., and Dandouras, I.: Average magnetotail electron and proton pitch angle distributions from Cluster PEACE and CIS observations, Geophys. Res. Lett., 38, L06103, doi:10.1029/2011GL046770, 2011.

Yao, Z. H., Pu, Z. Y., Fu, S. Y., Angelopoulos, V., Kubyshkina, M., Xing, X., Lyons, L., Nishimura, Y., Xie, L., Wang, X. G., Xiao, C. J., Cao, X., Liu, J., Zhang, H., Nowada, M., Zong, Q. G., Guo, R. L., Zhong, J., and Li, J. X.: Mechanism of substorm current wedge formation: THEMIS observations, Geophys. Res. Lett., 39, L13102, doi:10.1029/2012GL052055, 2012.

Yao, Z. H., Sun, W. J., Fu, S. Y., Pu, Z. Y., Liu, J., Angelopoulos, V., Zhang, X. J., Chu, X. N., Shi, Q. Q., Guo, R. L., and Zong, Q. G.: Current structures associated with dipolarization fronts, J. Geophys. Res.-Space, 118, 6980-6985, 2013.
Yao, Z. H., Pu, Z. Y., Du, A. M., Angelopoulos, V., Owen, C. J., Liu, J., Chu, X. N., Cao, X., Fu, S. Y., Zong, Q. G., and Wang, Y.: Pressure gradient evolution in the near-Earth magnetotail at the arrival of BBFs, Chinese Sci. Bull., 59, 4804-4808, 2014a.

Yao, Z. H., Pu, Z. Y., Owen, C. J., Fu, S. Y., Chu, X. N., Liu, J., Angelopoulos, V., Rae, I. J., Yue, C., Zhou, X. Z., Zong, Q. G., Cao, X., Shi, Q. Q., Forsyth, C., and Du, A. M.: Current reduction in a pseudo-breakup event: THEMIS observations, J. Geophys. Res.-Space, 119, 8178-8187, 2014b.

Zhang, X. and Angelopoulos, V.: On the relationship of electrostatic cyclotron harmonic emissions with electron injections and dipolarization fronts, J. Geophys. Res.-Space, 119, 2536-2549, doi:10.1002/2013JA019540, 2014.

Zhou, M., Ashour-Abdalla, M., Deng, X., Schriver, D., ElAlaoui, M., and Pang, Y.: THEMIS observation of multiple dipolarization fronts and associated wave characteristics in the near-Earth magnetotail, Geophys. Res. Lett., 36, L20107, doi:10.1029/2009GL040663, 2009.

Zhou, X. Z., Angelopoulos, V., Liu, J., Runov, A., and Li, S. S.: On the origin of pressure and magnetic perturbations ahead of dipolarization fronts, J. Geophys. Res.-Space, 119, 211-220, doi:10.1002/2013JA019394, 2014. 